\title{
Analysis of Nonuniform Transmission Lines With an Iterative and Adaptive Perturbation Technique
}

\author{
Paolo Manfredi, Member, IEEE, Daniël De Zutter, Fellow, IEEE, Dries Vande Ginste, Senior Member, IEEE.
}

\begin{abstract}
This paper presents an iterative and adaptive perturbation technique for the analysis of nonuniform transmission lines. Place-dependent variations of the per-unit-length parameters are interpreted as perturbations with respect to their average values along the line. This allows casting the governing equations for the corresponding perturbations of the voltages and currents as those of a uniform transmission line with distributed sources. Therefore, standard transmission line theory is used to calculate these perturbation terms. Specifically, perturbations of increasing order are computed iteratively starting from the solution of the unperturbed line. The accuracy is adaptively adjusted by setting a threshold on the convergence of the solution. The algorithm turns out to be simple to implement and very accurate, yet faster than traditional approaches based on the discretization of the line into uniform subsections. The technique is validated through the analysis of several nonuniform transmission line structures of relevance in EMC applications, namely uniformly and nonuniformly twisted wire pairs as well as a cable bundle with lacing cords.
\end{abstract}

Index Terms-Cable bundles, crosstalk, multiconductor transmission lines, nonuniform transmission lines, transmission line theory, twisted wire pairs.

\section{INTRODUCTION}

Nonuniform transmission lines (NUTLs) are widely encountered in several transport and industrial applications and their study still represents an active field of research in EMC [1][5]. One of the most common examples are twisted wire pairs (TWPs), which are used to transfer information in communication systems due to their high immunity to both conducted [6] and radiated [7] electromagnetic interference. Many other contributions addressed the EMC modeling of the TWP performance in terms of susceptibility to external fields [8]-[11] or wave propagation and crosstalk [12]-[16]. A second example of a NUTL is a hand-assembled cable bundle, where the (random) meandering of wires yields a geometry far from being uniform along the line [17]-[19]. Additionally, cable bundles may assume a nonuniform geometry as a result of the presence of lacing cords, which are used to hold wires together in long harnesses [20].

Whereas the theory for uniform transmission lines is well established [21], the analysis of NUTLs remains a research topic, as no closed-form solution exists except for some special cases [22]-[24]. The classical approach to tackle this problem is to subdivide the line into locally uniform sections and to analyze them upon concatenation of their chain-parameter

This work was funded by the Research Foundation Flanders (FWOVlaanderen). P. Manfredi is currently an FWO Postdoctoral Research Fellow.

P. Manfredi, D. De Zutter and D. Vande Ginste are with the Electromagnetics Group, Department of Information Technology, Ghent University/iMinds, 9000 Gent, Belgium (e-mail: paolo.manfredi@ugent.be, daniel.dezutter@ugent.be, dries.vandeginste@ugent.be). matrix [21] or ABCD matrix [25]. A general rule for the number of discretizations to be used is missing, yet it has been shown that, for instance, as many as 128 discretization per twist are required to accurately capture the response of a TWP [15]. This can lead to large computational times, especially in those cases that require a repeated-run analysis to provide a statistical description of problems that are inherently stochastic (see, e.g., [17]-[19] and [26]-[28]). Other approaches subdivide the line into nonuniform sections of exponential [29] or linear [30] type, for which analytical solutions exist, but limited to the case of lossless lines with frequency-independent per-unit-length (p.u.l.) parameters.

There exist other techniques, mainly proposed for microwave structures, that are based on several different solutions, including the Taylor series expansion of the p.u.l. parameters [2], finite-difference time domain methods [3], [5], Chebyshev interpolations [31], [32], waveform relaxation [33], the method of characteristics [34], full-wave simulations [35], rational approximations [36], wavelets theory [37], [38], the differential quadrature method [39], or congruence transforms [40]. These methods, however, rely on specific assumptions, idealizations or approximations, and they often turn out to be rather time consuming and/or cumbersome to implement.

An alternative approach converts the NUTL equations into the equations of uniform lines excited by equivalent distributed sources [41]. This method, originally presented for single lines, has been recently recast in terms of a two-step perturbation technique applied to single or differential [42] as well as to arbitrary multiconductor transmission lines [43]. The nonuniformity is in this case interpreted as a perturbation with respect to an average uniform line. A perturbation is then also assumed for the voltages and currents along the line. Lengthy manipulations of the governing equations allow to arrive at semi-analytical expressions for the first- and secondorder perturbations of the voltages and currents. Nevertheless, the complexity of these equations discourages to develop analogous results for higher-order terms.

So far, the aforementioned method has been applied to printed circuit board interconnects, in which the spatial variation of the p.u.l. parameters is limited by geometrical constraints, thus rendering the second-order perturbation sufficiently accurate [42]-[44]. On the contrary, the variations of wire positions in TWPs or in nonuniform cable harnesses, as considered in this paper, result in a more severe variation of the p.u.l. parameters (especially, the capacitance) along the line. In these cases, a second-order perturbation no longer suffices. To accurately describe all relevant phenomena such as, e.g., shielding effects between wires, many higher-order perturbations need to be taken into account. 
The aim of this paper is therefore to generalize the technique presented in [43] to an arbitrary number of perturbation steps. However, instead of attempting to derive unmanageable closed-form expressions, an iterative procedure is put forward. The governing equations for the perturbations of the voltages and currents are expressed as the equations of a uniform transmission line with distributed sources that depend on the previous perturbation step. Next, the equations are iteratively solved via the well-known and standard transmission line theory, in analogy with the problem of electromagnetic field illumination of the line [21]. The iteration is adaptively stopped when the result has converged within a predefined threshold. The methodology is general and applies to arbitrary multiconductor NUTLs with frequency-dependent parameters. Moreover, it is simple and straightforward to implement, being nonetheless very fast and accurate. The frequency-domain solution is readily combined with a scattering characterization to obtain time-domain results [45]. Applications to nonuniform cables are provided to validate the proposed technique.

The remainder of the paper is organized as follows. The proposed perturbation method is outlined in Section II. Illustrative application examples and validations are provided in Section III. The achieved results are discussed in Section IV. Finally, a summary and conclusions are presented in Section $\mathrm{V}$.

\section{Iterative Perturbation Technique}

\section{A. NUTL Equations}

Consider the frequency-domain Telegrapher's equations for an $N$-conductor NUTL of length $\ell$ :

$$
\begin{aligned}
& \frac{d}{d z} \mathbf{V}(z, \omega)=-j \omega \mathcal{L}(z, \omega) \mathbf{I}(z, \omega) \\
& \frac{d}{d z} \mathbf{I}(z, \omega)=-j \omega \mathcal{C}(z, \omega) \mathbf{V}(z, \omega),
\end{aligned}
$$

where $z \in[0, \ell]$ denotes the longitudinal coordinate, $\mathbf{V}$ and $\mathbf{I}$ are $N$-vectors collecting the voltages and currents along the line, and $\mathcal{L}$ and $\mathcal{C}$ are the $N \times N$ complex and place-dependent p.u.l. inductance and capacitance matrices, defined as

$$
\begin{aligned}
& \mathcal{L}(z, \omega)=\mathbf{L}(z, \omega)+\frac{\mathbf{R}(z, \omega)}{j \omega} \\
& \mathcal{C}(z, \omega)=\mathbf{C}(z, \omega)+\frac{\mathbf{G}(z, \omega)}{j \omega},
\end{aligned}
$$

encompassing the frequency-dependent p.u.l. resistance, inductance, conductance and capacitance matrices of the line. From now on, the dependence of the voltages, currents and p.u.l. parameters on the angular frequency $\omega$ is dropped for notational convenience. These quantities are understood to be referred to a given frequency.

\section{B. Perturbation of Voltages, Currents and p.u.l. Parameters}

The $z$-dependent p.u.l. parameters are now represented in terms of a uniform (i.e., constant) component plus a placedependent variation:

$$
\begin{aligned}
& \mathcal{L}(z)=\tilde{\mathcal{L}}+\Delta \mathcal{L}(z) \\
& \mathcal{C}(z)=\tilde{\mathcal{C}}+\Delta \mathcal{C}(z),
\end{aligned}
$$

with the constant part being the average of the matrix values over the line length, i.e.,

$$
\begin{aligned}
& \tilde{\mathcal{L}}=\frac{1}{\ell} \int_{0}^{\ell} \mathcal{L}(z) d z \\
& \tilde{\mathcal{C}}=\frac{1}{\ell} \int_{0}^{\ell} \mathcal{C}(z) d z,
\end{aligned}
$$

whereas $\Delta \mathcal{L}(z)$ and $\Delta \mathcal{C}(z)$ are the variations (or perturbations) of the p.u.l. inductance and capacitance, respectively, that remain after subtraction of the constant part. The above decomposition is analogous to the one applied to the modal formulation of uniform differential lines in [46], where the aim was to assess the effect of unintentional geometrical imbalance.

The voltages and currents are represented as

$$
\begin{aligned}
& \mathbf{V}(z)=\mathcal{V}_{0}(z)+\sum_{k=1}^{K} \mathcal{V}_{k}(z) \\
& \mathbf{I}(z)=\mathcal{I}_{0}(z)+\sum_{k=1}^{K} \mathcal{I}_{k}(z)
\end{aligned}
$$

i.e., as the summation of unperturbed values $\mathcal{V}_{0}, \mathcal{I}_{0}$ and perturbations $\mathcal{V}_{k}, \mathcal{I}_{k}$ of increasing order. Note that in [42] and [43], only the case $K=2$ was considered, for which closedform expressions could be provided for $\mathcal{V}_{1}, \mathcal{I}_{1}, \mathcal{V}_{2}$ and $\mathcal{I}_{2}$.

Substituting (3) and (5) into (1) and collecting the terms of the same order, yields

$$
\begin{aligned}
& \frac{d}{d z} \mathcal{V}_{0}(z)=-j \omega \tilde{\mathcal{L}} \mathcal{I}_{0}(z) \\
& \frac{d}{d z} \mathcal{I}_{0}(z)=-j \omega \tilde{\mathcal{C}} \mathcal{V}_{0}(z)
\end{aligned}
$$

and

$$
\begin{aligned}
& \frac{d}{d z} \mathcal{V}_{k}(z)=-j \omega \tilde{\mathcal{L}} \mathcal{I}_{k}(z)+\mathcal{V}_{F, k}(z) \\
& \frac{d}{d z} \mathcal{I}_{k}(z)=-j \omega \tilde{\mathcal{C}} \mathcal{V}_{k}(z)+\mathcal{I}_{F, k}(z)
\end{aligned}
$$

$k=1, \ldots, K$, with the equivalent distributed sources defined as

$$
\begin{aligned}
& \mathcal{V}_{F, k}(z)=-j \omega \Delta \mathcal{L}(z) \mathcal{I}_{k-1}(z) \\
& \mathcal{I}_{F, k}(z)=-j \omega \Delta \mathcal{C}(z) \mathcal{V}_{k-1}(z) .
\end{aligned}
$$

It is worth noting that:

- The governing equations (6) for the zeroth-order component of the voltages and currents are those of a uniform transmission line with constant p.u.l. parameters $\tilde{\mathcal{L}}$ and $\tilde{\mathcal{C}}$.

- The governing equations (7) for the $k$ th-order perturbation of the voltages and currents are those of the same uniform line with additional distributed sources.

- According to (8), such distributed sources depend on the previous solution obtained for $k-1$.

\section{Solution Procedure}

The explicit, general solution of (7) is given in [21] for the illumination of a uniform transmission line by an external 
electromagnetic field:

$$
\begin{aligned}
& \mathcal{V}_{k}(z)=\boldsymbol{\Phi}_{11}(z) \mathcal{V}_{k}(0)+\boldsymbol{\Phi}_{12}(z) \mathcal{I}_{k}(0)+\mathcal{V}_{F T, k}(z) \\
& \mathcal{I}_{k}(z)=\boldsymbol{\Phi}_{21}(z) \mathcal{V}_{k}(0)+\boldsymbol{\Phi}_{22}(z) \mathcal{I}_{k}(0)+\mathcal{I}_{F T, k}(z)
\end{aligned}
$$

with $\boldsymbol{\Phi}_{i j}(i, j=1,2)$ the blocks of the chain-parameter matrix, given by

$$
\begin{aligned}
& \boldsymbol{\Phi}_{11}(z)=\frac{1}{2} \mathbf{Y}^{-1} \mathbf{T}\left(\mathbf{e}^{\gamma z}+\mathbf{e}^{-\gamma z}\right) \mathbf{T}^{-1} \mathbf{Y} \\
& \boldsymbol{\Phi}_{12}(z)=-\frac{1}{2} \mathbf{Y}^{-1} \mathbf{T} \boldsymbol{\gamma}\left(\mathbf{e}^{\gamma z}-\mathbf{e}^{-\gamma z}\right) \mathbf{T}^{-1} \\
& \boldsymbol{\Phi}_{21}(z)=-\frac{1}{2} \mathbf{T}\left(\mathbf{e}^{\gamma z}-\mathbf{e}^{-\gamma z}\right) \boldsymbol{\gamma}^{-1} \mathbf{T}^{-1} \mathbf{Y} \\
& \boldsymbol{\Phi}_{22}(z)=\frac{1}{2} \mathbf{T}\left(\mathbf{e}^{\gamma z}+\mathbf{e}^{-\gamma z}\right) \mathbf{T}^{-1}
\end{aligned}
$$

where $\mathbf{Y}=j \omega \tilde{\mathcal{C}}$ and the matrices $\mathbf{T}$ and $\gamma$ are given by the eigendecomposition

$$
\mathbf{T}^{-1} \mathbf{Y Z T}=\gamma^{2}
$$

with $\mathbf{Z}=j \omega \tilde{\mathcal{L}}$. Furthermore, the so-called forcing terms $\mathcal{V}_{F T, k}$ and $\mathcal{I}_{F T, k}$ are given by

$$
\begin{aligned}
& \mathcal{V}_{F T, k}(z)= \\
& \quad \int_{0}^{z} \boldsymbol{\Phi}_{11}(z-\tau) \mathcal{V}_{F, k}(\tau) d \tau+\int_{0}^{z} \boldsymbol{\Phi}_{12}(z-\tau) \mathcal{I}_{F, k}(\tau) d \tau \\
& \mathcal{I}_{F T, k}(z)= \\
& \quad \int_{0}^{z} \boldsymbol{\Phi}_{21}(z-\tau) \mathcal{V}_{F, k}(\tau) d \tau+\int_{0}^{z} \boldsymbol{\Phi}_{22}(z-\tau) \mathcal{I}_{F, k}(\tau) d \tau .
\end{aligned}
$$

Finally, $\mathcal{V}_{k}(0)$ and $\mathcal{I}_{k}(0)$ are the solution at the near-end termination, which is calculated by incorporating the boundary conditions, as discussed in the next section. The expression (9) is valid also for (6), i.e., for $k=0$, for which however the forcing terms (12) vanish due to the absence of distributed sources.

The calculation of the forcing terms (12) involves convolution integrals. Yet, these convolutions are reduced to standard integrals thanks to the exponential dependence of the chainparameter matrix on $z$. In fact, substitution of (10) into (12) allows to rewrite the latter as

$$
\begin{aligned}
\mathcal{V}_{F T, k}(z)= & +\frac{1}{2} \mathbf{Y}^{-1} \mathbf{T} \mathbf{e}^{\gamma z} \int_{0}^{z} \mathbf{e}^{-\gamma \tau} \mathbf{T}^{-1} \mathbf{Y} \mathcal{V}_{F, k}(\tau) d \tau \\
& +\frac{1}{2} \mathbf{Y}^{-1} \mathbf{T} \mathbf{e}^{-\gamma z} \int_{0}^{z} \mathbf{e}^{\gamma \tau} \mathbf{T}^{-1} \mathbf{Y} \mathcal{V}_{F, k}(\tau) d \tau \\
& -\frac{1}{2} \mathbf{Y}^{-1} \mathbf{T} \gamma \mathbf{e}^{\gamma z} \int_{0}^{z} \mathbf{e}^{-\gamma \tau} \mathbf{T}^{-1} \mathcal{I}_{F, k}(\tau) d \tau \\
& +\frac{1}{2} \mathbf{Y}^{-1} \mathbf{T} \gamma \mathbf{e}^{-\gamma z} \int_{0}^{z} \mathbf{e}^{\gamma \tau} \mathbf{T}^{-1} \mathcal{I}_{F, k}(\tau) d \tau \\
\mathcal{I}_{F T, k}(z)= & -\frac{1}{2} \mathbf{T} \mathbf{e}^{\gamma z} \int_{0}^{z} \mathbf{e}^{-\gamma \tau} \boldsymbol{\gamma}^{-1} \mathbf{T}^{-1} \mathbf{Y} \mathcal{V}_{F, k}(\tau) d \tau \\
& +\frac{1}{2} \mathbf{T} \mathbf{e}^{-\gamma z} \int_{0}^{z} \mathbf{e}^{\gamma \tau} \boldsymbol{\gamma}^{-1} \mathbf{T}^{-1} \mathbf{Y} \mathcal{V}_{F, k}(\tau) d \tau \\
& +\frac{1}{2} \mathbf{T e}^{\gamma z} \int_{0}^{z} \mathbf{e}^{-\gamma \tau} \mathbf{T}^{-1} \mathcal{I}_{F, k}(\tau) d \tau \\
& +\frac{1}{2} \mathbf{T e}^{-\gamma z} \int_{0}^{z} \mathbf{e}^{\gamma \tau} \mathbf{T}^{-1} \mathcal{I}_{F, k}(\tau) d \tau
\end{aligned}
$$

The above formulation allows a more efficient calculation of the cumulative integrals, as needed to evaluate the forcing terms along $z$. These are in turn necessary to evaluate the solution (9) for the update of the distributed sources (8).

It should be noted that the matrix blocks (10), as well as the matrices $\mathbf{Y}, \gamma$ and $\mathbf{T}$, refer only to the uniform line parameters $\tilde{\mathcal{L}}$ and $\tilde{\mathcal{C}}$, and they are therefore calculated only once per frequency point.

\section{Incorporation of the Terminal Conditions}

In order to fully determine the solution (9) of the equations (6) and (7), $\mathcal{V}_{k}(0)$ and $\mathcal{I}_{k}(0)$, with $k=0, \ldots, K$, need to be found, which requires proper boundary conditions. These are typically given as the Thévenin or Norton equivalents of the line terminations [21]. For example, considering the Thévenin representation with equivalent voltage sources $\mathbf{V}_{S, L}$ and impedances $\mathbf{Z}_{S, L}$ at the source (S) (or near-end, $z=0$ ) and load (L) (or far-end, $z=\ell$ ) terminations, the boundary conditions read [43]

$$
\begin{aligned}
& \mathcal{V}_{0}(0)=\mathbf{V}_{S}-\mathbf{Z}_{S} \mathcal{I}_{0}(0) \\
& \mathcal{V}_{0}(\ell)=\mathbf{V}_{L}+\mathbf{Z}_{L} \mathcal{I}_{0}(\ell),
\end{aligned}
$$

for (6), and

$$
\begin{aligned}
& \mathcal{V}_{k}(0)=-\mathbf{Z}_{S} \mathcal{I}_{k}(0) \\
& \mathcal{V}_{k}(\ell)=\mathbf{Z}_{L} \mathcal{I}_{k}(\ell),
\end{aligned}
$$

for (7). Briefly speaking, the original terminal excitation of the line appears only once, i.e., in the unperturbed solution.

Given the terminal conditions (14) and (15), the currents at $z=0$ are now readily derived as:

$$
\mathcal{I}_{0}(0)=\mathbf{A}^{-1}\left[\left(\boldsymbol{\Phi}_{11}(\ell)-\mathbf{Z}_{L} \boldsymbol{\Phi}_{21}(\ell)\right) \mathbf{V}_{S}-\mathbf{V}_{L}\right]
$$

for the unperturbed solution and

$$
\mathcal{I}_{k}(0)=\mathbf{A}^{-1}\left[\mathcal{V}_{F T, k}(\ell)-\mathbf{Z}_{L} \mathcal{I}_{F T, k}(\ell)\right]
$$


for the perturbation terms, with

$$
\mathbf{A}=\left[\boldsymbol{\Phi}_{11}(\ell) \mathbf{Z}_{S}+\mathbf{Z}_{L} \boldsymbol{\Phi}_{22}(\ell)-\boldsymbol{\Phi}_{12}(\ell)-\mathbf{Z}_{L} \boldsymbol{\Phi}_{21}(\ell) \mathbf{Z}_{S}\right] .
$$

The voltages $\mathcal{V}_{k}(0)$ at the near-end termination are found by substitution of (16) and (17) into (14) and (15).

\section{E. Iterative and Adaptive Solution}

Based on the information collected in the previous sections, the following iterative procedure is put forward for the simulation of a NUTL. First, the unperturbed solution $\mathcal{V}_{0}$ and $\mathcal{I}_{0}$ of the line (6) with averaged p.u.l. parameters (4) is calculated using the chain-parameter matrix (10) and the proper boundary conditions. Next, the first-order equivalent distributed sources $\mathcal{V}_{F, 1}$ and $\mathcal{I}_{F, 1}$ are calculated with (8), based on the place-dependent variation of the p.u.l. parameters. The first-order perturbations $\mathcal{V}_{1}$ and $\mathcal{I}_{1}$ are computed as the solution of (7), after incorporating the pertinent forcing terms (13) into the terminal conditions, as indicated in Section II-D. The second-order equivalent distributed sources $\mathcal{V}_{F, 2}$ and $\mathcal{I}_{F, 2}$ are then calculated, and the above procedure is iterated until the solution has converged below a predefined threshold.

Specifically, at each iteration it is checked whether the relative contribution provided by the last perturbation (i.e., the last computed perturbation term over the sum of all available terms) is sufficiently small in magnitude. In mathematical terms, at a given frequency, the solution is stopped at iteration $K$ if

$$
D_{\max }=\max \left(\left|\frac{\mathcal{V}_{K}(z)}{\sum_{k=0}^{K} \mathcal{V}_{k}(z)}\right|,\left|\frac{\mathcal{I}_{K}(z)}{\sum_{k=0}^{K} \mathcal{I}_{k}(z)}\right|\right)<\delta_{\mathrm{th}},
$$

where the maximum is intended over all the elements of $\mathcal{V}$ and $\mathcal{I}$. Either the values along the entire line length, or those at the terminations only, may be considered depending on the quantities of interest.

As yet, no rigorous proof could be found as to the guaranteed convergence of the series (5). However, for all practical engineering purposes investigated so far, and reported in this paper, this turns out to be the case.

\section{NumERICAL RESUlTS AND APPLICATIONS}

The proposed technique has been implemented in MATLAB and it is applied in this section to the analysis of nonuniform cable lines, containing either TWPs or lacing cords. Reference results are generated by discretizing the line into uniform cascaded sections (UCS) [21], [25]. As dielectric coatings are included, the method in [47] and [48] is used to calculate the p.u.l. capacitance and inductance matrices from the cable cross-section at a given position along the line. For the perturbation technique, a cumulative Simpson's quadrature is adopted for the calculation of the integrals (13), and the threshold for the adaptive solution is set to $\delta_{\text {th }}=1 \%$. The simulations are performed on an ASUS U30S laptop with an Intel(R) Core(TM) i3-2330M, CPU running at $2.20 \mathrm{GHz}$ and 4 GB of RAM.

\section{A. Uniformly Twisted Pair}

The first example is taken from [15] and deals with the coupling between a TWP and a straight wire (see Fig. 1). The wires have a total diameter $d=1.7 \mathrm{~mm}$ and a $0.11-\mathrm{mm}$ thick PVC coating (relative permittivity of 3.5). The separation between the straight wire and the center of the TWP is $s=$ $2.55 \mathrm{~mm}$, so the three wires are touching when they are lined up horizontally, and they lie at a height $h=5 \mathrm{~cm}$ above a perfect ground plane. The line has a length $\ell=1 \mathrm{~m}$ and the TWP has $N=25$ full twists. The wires are terminated as shown in Fig. 1(b).

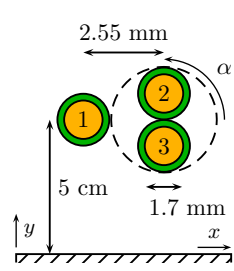

(a)

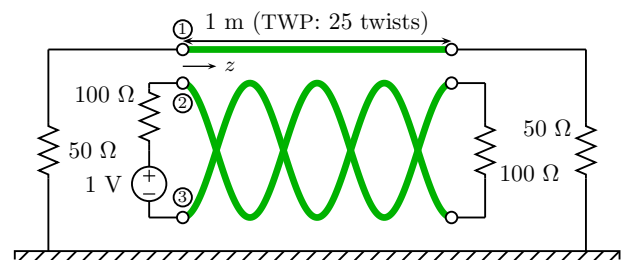

(b)
Fig. 1. Line configuration of a straight wire and a uniformly twisted pair: cross-sectional view at $z=0$ (a) and longitudinal view with terminations (b).

The place-dependent cross-sectional $x$-y-coordinates of the twisted wires \#2 and \#3 are given by

$$
\begin{aligned}
& x_{2}(z)=s+(d / 2) \cos (\varphi(z)) \\
& y_{2}(z)=h+(d / 2) \sin (\varphi(z)) \\
& x_{3}(z)=s-(d / 2) \cos (\varphi(z)) \\
& y_{3}(z)=h-(d / 2) \sin (\varphi(z)),
\end{aligned}
$$

with $\varphi(z)=\alpha+2 \pi z N / \ell$, and where the angle $\alpha$ determines the position of the TWP at $z=0$. The position of wire \#1 is fixed in the $x y$-plane with $x_{1}=0$ and $y_{1}=5 \mathrm{~cm}$. At a given longitudinal position $z$, the location of the TWP, and hence, the entire cross-section, is fully determined by the rotation angle $\varphi(z)$.

In order to remove the overhead of the calculation of the p.u.l. parameters in the following simulations, a spline interpolation of the inductance and capacitance matrices is constructed as a function of $\varphi$. An interpolation over 200 points yields a maximum relative error well below $0.1 \%$. The average inductance and capacitance matrices along the line are

$$
\tilde{\mathcal{L}}=\left[\begin{array}{lll}
936.6 & 739.7 & 739.7 \\
739.7 & 915.3 & 808.7 \\
739.7 & 808.7 & 915.3
\end{array}\right] \mathrm{nH} / \mathrm{m}
$$

and

$$
\tilde{\mathcal{C}}=\left[\begin{array}{rrr}
51.7 & -23.1 & -23.1 \\
-23.1 & 107.1 & -79.8 \\
-23.1 & -79.8 & 107.1
\end{array}\right] \mathrm{pF} / \mathrm{m},
$$

respectively, whereas the maximum relative perturbations of the matrix entries over the line length are $|\Delta \mathcal{L} / \tilde{\mathcal{L}}|_{\max }=8 \%$ and $|\Delta \mathcal{C} / \tilde{\mathcal{C}}|_{\max }=251 \%$. Whilst the longitudinal variation of the p.u.l. inductance is relatively small, the variation of the capacitance matrix is extremely high due to the mutual shielding of wires \#2 and \#3 with respect to wire \#1.

Fig. 2 shows the magnitude of the current on the straight wire along the first full twist of the TWP, at a frequency of 


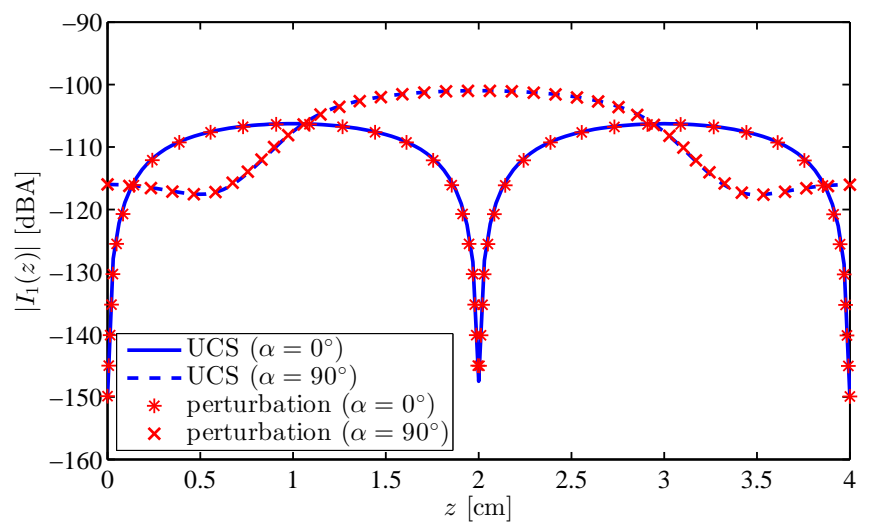

Fig. 2. Magnitude of the current along the straight wire at $11 \mathrm{MHz}$. Lines: results computed with the UCS method for $\alpha=0^{\circ}$ (solid) and $\alpha=90^{\circ}$ (dashed); markers: same results obtained with the proposed perturbation technique.

$11 \mathrm{MHz}$. The reference results are computed with the UCS approach for two starting positions of the TWP, corresponding to $\alpha=0^{\circ}$ (solid line) and $\alpha=90^{\circ}$ (dashed line). Each twist has been discretized into 128 uniform sections. There is a strong impact of the starting position on the current magnitude, as already observed in [15]. The same results are computed via the proposed perturbation technique (markers) with up to 3 iterations, showing excellent agreement.

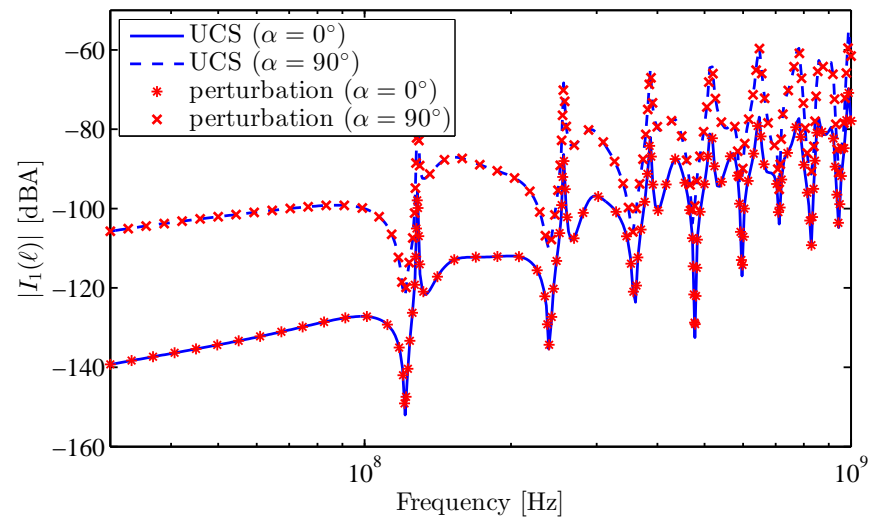

Fig. 3. Magnitude versus frequency of the current at the far-end termination of the straight wire. Curve identification is as in the caption of Fig. 2.

In addition, Fig. 3 shows the magnitude of the current at the far-end termination of the straight wire over 300 frequency points from $300 \mathrm{MHz}$ to $1 \mathrm{GHz}$. Also for this analysis, remarkable agreement is established between the reference UCS method and the proposed perturbation technique. The number of perturbation steps used by the latter varies with the frequency, and ranges from 2 to 10 in order to achieve $D_{\max }<1 \%$. Hence, a two-step perturbation as proposed in [42] and [43] is not always accurate enough for this line. The average number of iterations per frequency is 3.5 for $\alpha=0^{\circ}$ and 2.6 for $\alpha=90^{\circ}$, leading to a simulation time of $7.2 \mathrm{~s}$ and $5.3 \mathrm{~s}$ in the two cases, respectively. The simulation time for the UCS is $6.5 \mathrm{~s}$. It should be noted that, in this case, the periodicity of the line allows an efficient implementation of the UCS approach. Nevertheless, even when exploiting this very idealized situation with the UCS, the perturbation technique has a comparable or even lower execution time.

\section{B. Randomly Twisted Pair}

In this second example, a stochastic twisting pattern is ascribed to the TWP of Fig. 1, using the model suggested in [7]. The configuration of the terminations remains the same. However, the rotation angle $\varphi(z)$ is now defined as

$$
\varphi(z)=\int_{0}^{z} \alpha\left(z^{\prime}\right) d z^{\prime}
$$

where

$$
\alpha(z)=\left[\left(\frac{d}{2}\right)^{2}+\left(\frac{p(z)}{2 \pi}\right)^{2}\right]^{-1 / 2}
$$

and $p(z)$ (twist pitch function) is a Gaussian stochastic process with a nominal value $p_{\text {nom }}$ and correlation function

$$
c\left(z, z^{\prime}\right)=\sigma^{2} e^{-\frac{\left|z-z^{\prime}\right|^{2}}{\rho^{2}}} .
$$

The parameters $\sigma$ and $\rho$ control the nonuniformity and deformation of the twisting. For the simulation, $p_{\text {nom }}=2 \mathrm{~cm}$, $\sigma / p_{\text {nom }}=0.3$ and $\rho / p_{\text {nom }}=0.5$ are considered [7].

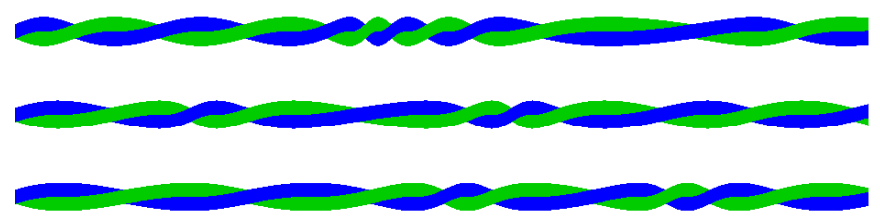

Fig. 4. Three profile realizations of the stochastic TWP over a 10-cm section (on scale).

Fig. 4 shows three different realizations (with axis aspect ratio preserved) of the randomly twisted pair over a length of $10 \mathrm{~cm}$. It is important to point out that the structure is no longer periodic. For a given realization of $\varphi(z)$, the same spline interpolation as generated for the previous example is used to retrieve the p.u.l. inductance and capacitance matrices.

Fig. 5 shows the voltage at the far-end termination of the straight wire, computed for ten realizations of the stochastic TWP with both the UCS method (solid lines) and the proposed perturbation technique (markers). In the former case, the line has to be discretized into 4000 uniform sections to achieve convergence, which leads to a simulation time of $180 \mathrm{~s}$ for each realization. In contrast, the perturbation technique only requires about $18 \mathrm{~s}$. A remarkable tenfold speed-up is therefore achieved with the proposed methodology. It is worth noting that the exact time depends on the number of perturbation steps used by the adaptive solution, which in turn varies with the frequency and the specific realization. A maximum number of 8 perturbations has been used to calculate the results in Fig. 5. Although not visible in Fig. 5, for the higher frequencies the results from the UCS and the present method remain identical. 


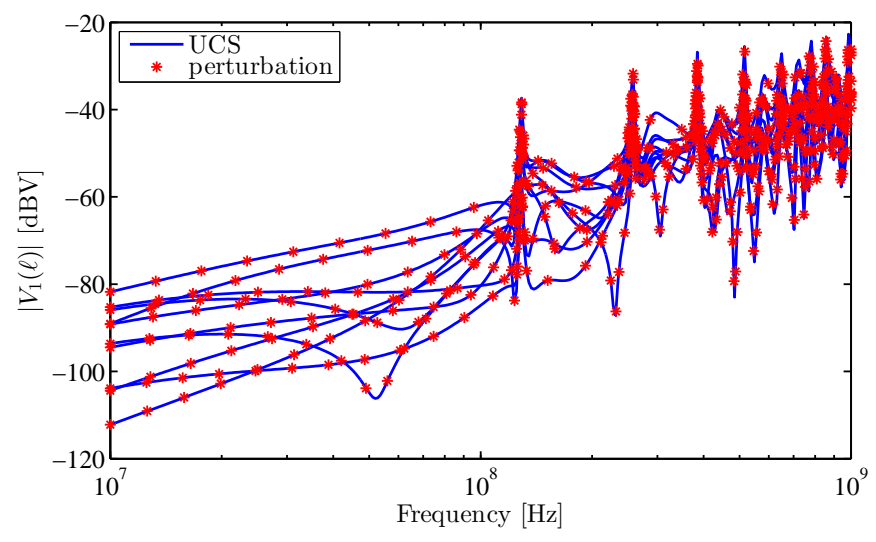

Fig. 5. Magnitude of the voltage at the far-end termination of the straight wire for ten realizations of the randomly twisted pair, computed with both the UCS approach (lines) and the perturbation technique (markers).

\section{Cable Bundle With Lacing Cords}

The final example considers a cable bundle with lacing cords, which are commonly used to hold wires together in long harnesses. The presence of lacing cords is modeled as proposed in [20] and illustrated in Fig. 6: at the beginning and end of the harness, as well as at the location of the lacing cords, the cable cross-section has a minimum radial dimension $r_{0}$. The cross-section radius expands between two lacing cords, reaching a maximum value $\alpha r_{0}$. This behavior is periodically repeated all over the cable length. Assuming that the cords are evenly spaced, the radial coordinate of each wire at a given longitudinal position $z$ is multiplied by a sinusoidal coefficient defined as

$$
\delta_{r}(z)=\frac{1}{2}(\alpha+1)+\frac{1}{2}(\alpha-1) \sin (2 \pi z / D-\pi / 2),
$$

where $D=\ell /\left(N_{L C}+1\right)$ is the lace-to-lace distance and $N_{L C}$ is the number of lacing cords. A spline interpolation of the p.u.l. parameters as a function of $\delta_{r}$ is created to efficiently sample the inductance and capacitance matrices in the simulations.

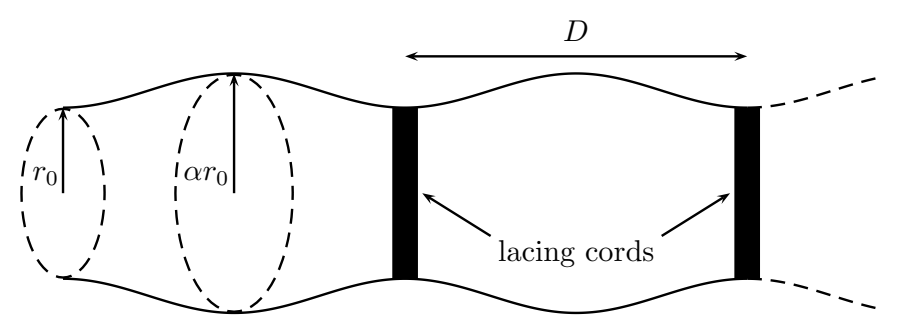

Fig. 6. Side view of a cable harness model with lacing cords [20].

The application refers to a 24-wire cable with the initial cross-section displayed in Fig. 7. The wires have a radius of $0.75 \mathrm{~mm}$. The radius of the dielectric coating is $1.15 \mathrm{~mm}$ and its relative permittivity is 2.6. The harness lies at a height of $1.8 \mathrm{~cm}$ above a PEC ground plane and it has $N_{L C}=19$ lacing cords over a length of $4 \mathrm{~m}$. The wires are terminated as indicated in Table I.

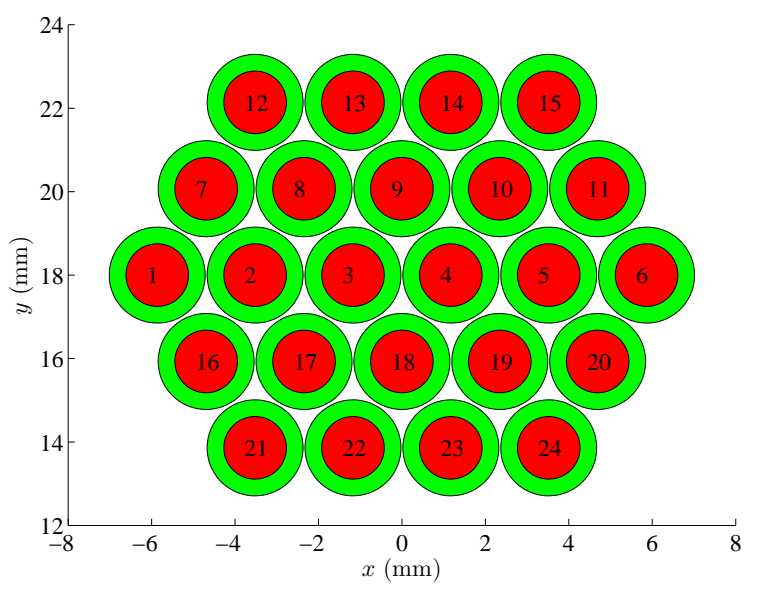

Fig. 7. Initial cross-section and wire numbering for the cable bundle with lacing cords.

In order to assess the impact of the expansion coefficient $\alpha$, its value is swept from 1 (uniform cable harness) to 3 . Fig. 8 shows the corresponding far-end crosstalk voltages produced on wires \#9 (dashed lines) and \#4 (solid lines) by the excitation of wire \#8 with a $1 \mathrm{~V}$ voltage source, and computed with the UCS technique by discretizing the line into 320 sections. The results computed with the perturbation technique (markers) agree once again very well. Intuitively, the crosstalk in principle reduces for larger values of $\alpha$, as this implies that the distance between the wires increases in between two lacing cords, thus reducing the overall coupling. Nonetheless, there exist some frequencies at which the crosstalk is higher for larger values of $\alpha$ (see, e.g., the region from 5 to $10 \mathrm{MHz}$ ). The simulation times are $107.6 \mathrm{~s}$ for the UCS method and $24.7 \mathrm{~s}$ for the perturbation technique, which uses a maximum number of 18 iterations, achieving a speed-up of $4.4 \times$.

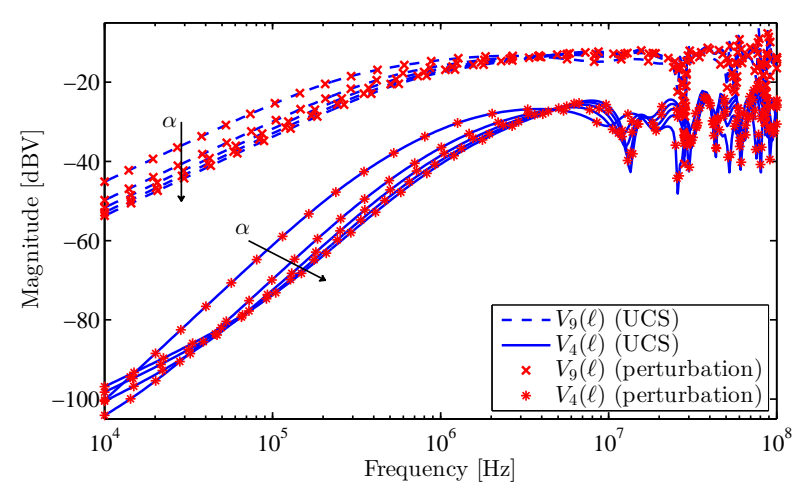

Fig. 8. Magnitude of the crosstalk voltage at the far-end terminations of wires \#9 and \#4, computed with both the UCS approach (dashed and solid lines) and the perturbation technique (markers) for $\alpha=1,1.5,2,2.5,3$.

\section{Discussion}

Fig. 9 shows the convergence of the maximum relative difference, given by (19), in the solution of the three con- 
TABLE I

RESISTIVE TERMINATIONS OF THE CABLES WITH LACING CORDS, IN OHMS.

\begin{tabular}{|c|c|c|c|c|c|c|c|c|c|c|c|c|c|c|c|c|c|c|c|c|c|c|c|c|}
\hline wire \# & 1 & 2 & 3 & 4 & 5 & 6 & 7 & 8 & 9 & 10 & 11 & 12 & 13 & 14 & 15 & 16 & 17 & 18 & 19 & 20 & 21 & 22 & 23 & 24 \\
\hline \hline near end & 10 & 100 & $5 \mathrm{k}$ & $10 \mathrm{k}$ & $100 \mathrm{k}$ & 100 & 100 & 50 & $1 \mathrm{k}$ & 10 & $100 \mathrm{k}$ & $10 \mathrm{k}$ & $1 \mathrm{k}$ & 10 & 100 & 50 & 100 & $100 \mathrm{k}$ & $10 \mathrm{k}$ & $1 \mathrm{k}$ & 10 & 500 & $1 \mathrm{k}$ & $1 \mathrm{k}$ \\
\hline far end & $100 \mathrm{k}$ & $50 \mathrm{k}$ & 50 & 300 & $6 \mathrm{k}$ & $10 \mathrm{k}$ & $1 \mathrm{k}$ & $2 \mathrm{k}$ & $20 \mathrm{k}$ & 800 & $4 \mathrm{k}$ & $7 \mathrm{k}$ & 800 & 40 & 20 & 600 & $3 \mathrm{k}$ & $4 \mathrm{k}$ & $100 \mathrm{k}$ & $1 \mathrm{k}$ & $2 \mathrm{k}$ & $20 \mathrm{k}$ & 30 & 50 \\
\hline
\end{tabular}

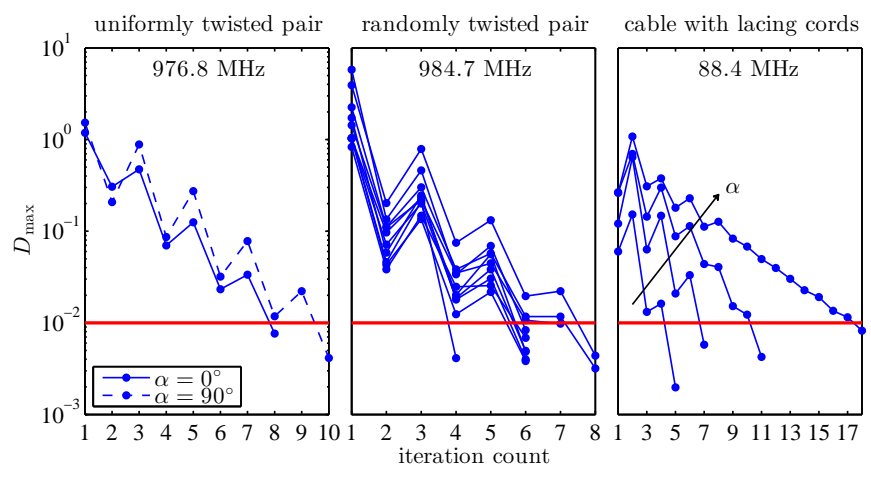

Fig. 9. Convergence of the maximum relative difference (19) for the three application examples. The iteration stop threshold of $1 \%$ is marked by the horizontal red line.

sidered application examples. It should be noted that this maximum difference is calculated over all the terminal voltages and currents of the line. For each case, the result refers to the frequency exhibiting the lowest convergence rate, i.e., 976.8 $\mathrm{MHz}$ for the uniformly twisted pair, 984.7 $\mathrm{MHz}$ for the nonuniformly twisted pair, and $88.4 \mathrm{MHz}$ for the cable with lacing cords. The $1 \%$ threshold that determines the convergence of the iterative solution is marked as a horizontal red line. The plots show that, in most of the situations, after two iterations the solution is still far from being converged. The results for the third example also highlight that, intuitively, the convergence becomes slower when the nonuniformity is higher, i.e., as the bundle expansion coefficient $\alpha$ increases. The result for $\alpha=1$, corresponding to a uniform cable harness, is not shown as the perturbation method immediately yields the exact solution.

Table II summarizes the computational times required to simulate the application examples, and the speed-up achieved by the proposed perturbation technique. It is interesting to note that the perturbation technique is faster despite requiring the numerical integration of the equivalent sources (13) over the line length. This is because it solves a uniform line, in which only the equivalent forcing terms need to be calculated and updated at each iteration. The cumulative integration is carried out in an efficient manner by exploiting the diagonal structure of the place-dependent matrix $\mathbf{e}^{ \pm \gamma z}$, thus allowing to achieve a substantial speed-up.

The largest speed-up is achieved in the analysis of the randomly twisted pair. This is the line that requires the largest amount of discretizations (4000) in the traditional UCS approach, thus suggesting that the proposed method is more efficient for highly nonuniform lines. Moreover, the analysis of the cable bundle shows that the performance of the perturbation technique scales well with the number of conductors, although the overall efficiency in this specific example is lower due to the small number of discretizations (320) required by the reference approach.

TABLE II

COMPUTATIONAL TIME AND SPEED-UP FOR THE CONSIDERED APPLICATION EXAMPLES.

\begin{tabular}{|c|r|r|r|}
\hline example & UCS approach & perturbation & speed-up \\
\hline \hline uniformly TWP $\left(\alpha=90^{\circ}\right)$ & $6.5 \mathrm{~s}$ & $5.3 \mathrm{~s}$ & $1.2 \times$ \\
\hline randomly TWP & $180.0 \mathrm{~s}$ & $18.0 \mathrm{~s}$ & $10 \times$ \\
\hline cable with lacing cords & $107.6 \mathrm{~s}$ & $24.7 \mathrm{~s}$ & $4.4 \times$ \\
\hline
\end{tabular}

\section{CONCLUSIONS}

This paper proposed an iterative and adaptive perturbation technique for the analysis of nonuniform multiconductor transmission lines. The solution of the NUTL equations is converted into the standard and well-known solution of uniform lines with equivalent distributed sources depending on the place-dependent variation of the p.u.l. parameters. An iterative solution allows to compute an arbitrary number of perturbations of the voltages and currents along the line. The simulation is stopped when the perturbation terms have converged within a given threshold. The technique is applied to the analysis of crosstalk in cables containing either TWPs or lacing cords. It turns to be very accurate, yet significantly faster than the standard solution based on the discretization of the line into uniform subsections.

\section{REFERENCES}

[1] H. Haase, T. Steinmetz, and J. Nitsch, "New propagation models for electromagnetic waves along uniform and nonuniform cables," IEEE Trans. Electromagn. Compat., vol. 46, no. 3, pp. 345-352, Aug. 2004.

[2] M. Khalaj-Amirhosseini, "Analysis of coupled nonuniform transmission lines using Taylor's series expansion," IEEE Trans. Electromagn. Compat., vol. 48, no. 3, pp. 594-600, Aug. 2006.

[3] M. Tang and J. Mao, "A precise time-step integration method for transient analysis of lossy nonuniform transmission lines," IEEE Trans. Electromagn. Compat., vol. 50, no. 1, pp. 166-174, Feb. 2008.

[4] G. Antonini, "Spectral models of lossy nonuniform multiconductor transmission lines," IEEE Trans. Electromagn. Compat., vol. 54, no. 2, pp. 474-481, Apr. 2012.

[5] K. Afrooz and A. Abdipour, "Efficient method for time-domain analysis of lossy nonuniform multiconductor transmission line driven by a modulated signal using FDTD technique," IEEE Trans. Electromagn. Compat., vol. 54, no. 2, pp. 482-494, Apr. 2012.

[6] F. Grassi and S. A. Pignari, "Immunity to conducted noise of data transmission along dc power lines involving twisted-wire pairs above ground," IEEE Trans. Electromagn. Compat., vol. 55, no. 1, pp. 195207, Feb. 2013.

[7] G. Spadacini and S. A. Pignari, "Numerical assessment of radiated susceptibility of twisted-wire pairs with random nonuniform twisting," IEEE Trans. Electromagn. Compat., vol. 55, no. 5, pp. 956-964, Oct. 2013. 
[8] C. D. Taylor and J. P. Castillo, "On the response of a terminated twistedwire cable excited by a plane-wave electromagnetic field," IEEE Trans. Electromagn. Compat., vol. 22, no. 1, pp. 16-19, Feb. 1980.

[9] J. G. Bredenson and H. Singh, "Response of a twisted pair excited by the nonuniform electromagnetic fields of nearby loops," IEEE Trans. Electromagn. Compat., vol. 24, no. 1, pp. 52-58, Feb. 1982.

[10] R. B. Armenta and C. D. Sarris, "Modeling the terminal response of a bundle of twisted-wire pairs excited by a plane wave," IEEE Trans. Electromagn. Compat., vol. 49, no. 4, pp. 901-913, Nov. 2007.

[11] G. Spadacini, F. Grassi, and S. A. Pignari, "Field-to-wire coupling model for the common mode in random bundles of twisted-wire pairs," IEEE Trans. Electromagn. Compat., vol. 57, no. 5, pp. 1246-1254, Oct. 2015.

[12] C. R. Paul and J. W. McKnight, "Prediction of crosstalk involving twisted pairs of wires-part I: a transmission-line model for twisted-wire pairs," IEEE Trans. Electromagn. Compat., vol. 21, no. 2, pp. 92-105, May 1979.

[13] K. Chamberlin, K. Komisarek, and K. Sivaprasad, "A method-ofmoments solution to the twisted-pair transmission line," IEEE Trans. Electromagn. Compat., vol. 37, no. 1, pp. 121-126, Feb. 1995.

[14] J. A. Brandão Faria and M. V. Guerreiro das Neves, "Analysis of the helical twisted-wire pair running above ground: transfer function evaluation," IEEE Trans. Electromagn. Compat., vol. 45, no. 2, pp. 449 453, May 2003.

[15] C. Jullien, P. Besnier, M. Dunand, and I. Junqua, "Advanced modeling of crosstalk between an unshielded twisted pair cable and an unshielded wire above a ground plane," IEEE Trans. Electromagn. Compat., vol. 55 , no. 1, pp. 183-194, Feb. 2013.

[16] A. Shoory, M. Rubinstein, A. Rubinstein, C. Romero, N. Mora, and F. Rachidi, "Application of the cascaded transmission line theory of Paul and McKnight to the evaluation of NEXT and FEXT in twisted wire pair bundles," IEEE Trans. Electromagn. Compat., vol. 55, no. 4, pp. 648-656, Aug. 2013.

[17] S. Salio, F. Canavero, D. Lecointe, and W. Tabbara, "Crosstalk prediction on wires bundles by Kriging approach,” in IEEE Int. Symp. Electromagn. Compat., Washington, DC, USA, Aug. 2000, vol. 1, pp. 197-202.

[18] S. Sun, G. Liu, J. L. Drewniak, and D. J. Pommerenke, "Hand-assembled cable bundle modeling for crosstalk and common-mode radiation prediction," IEEE Trans. Electromagn. Compat., vol. 49, no. 3, pp. 708-718, Aug. 2007.

[19] M. Wu, D. G. Beetner, T. H. Hubing, H. Ke, and S. Sun, "Statistical prediction of "reasonable worst-case" crosstalk in cable bundles," IEEE Trans. Electromagn. Compat., vol. 51, no. 3, pp. 842-851, Aug. 2009.

[20] D. Bellan and S. A. Pignari, "Efficient estimation of crosstalk statistics in random wire bundles with lacing cords," IEEE Trans. Electromagn. Compat., vol. 53, no. 1, pp. 209-218, Feb. 2011.

[21] C. R. Paul, Analysis of Multiconductor Transmission Lines, New York: Wiley, 1994.

[22] J. Nitsch and F. Gronwald, "Analytical solutions in nonuniform multiconductor transmission line theory," IEEE Trans. Electromagn. Compat. vol. 41, no. 4, pp. 469-479, Nov. 1999.

[23] A. Cheldavi, "Exact analysis of coupled nonuniform transmission lines with exponential power law characteristic impedance," IEEE Trans. Microw. Theory Techn., vol. 49, no. 1, pp. 197-199, Jan. 2001.

[24] C. L. Edwards, M. L. Edwards, S. Cheng, R. K. Stilwell, and C. C. Davis, "A simplified analytic CAD model for linearly tapered microstrip lines including losses," IEEE Trans. Microw. Theory Techn., vol. 52, no. 3, pp. 823-830, Mar. 2004

[25] J.-F. Mao and Z.-F. Li, "Analysis of the time response of nonuniform multiconductor transmission lines with a method of equivalent cascaded network chain," IEEE Trans. Microw. Theory Techn., vol. 40, no. 5, pp. 948-954, May 1992.

[26] A. Ciccolella and F. G. Canavero, "Stochastic prediction of wire coupling interference," in IEEE Int. Symp. Electromagn. Compat., Atlanta, GA, USA, Aug. 1995, pp. 51-56.

[27] M. Gonser, C. Keller, J. Hansen, and R. Weigel, "Advanced simulations of automotive EMC measurement setups using stochastic cable bundle models," in Asia-Pacific Symp. Electromagn. Compat., Beijing, China, Apr. 2010, pp. 590-593.

[28] C. Jullien, P. Besnier, M. Dunand, and I. Junqua, "Crosstalk analysis in complex aeronautical bundle," in IEEE Int. Symp. Electromagn. Compat., Brugge, Belgium, Sep. 2013, pp. 253-258.

[29] E. A. Oufi, A. S. Alfuhaid, and M. M. Saied, "Transient analysis of lossless single-phase nonuniform transmission lines," IEEE Trans. Power Del., vol. 9, no. 3, pp. 1694-1700, Jul. 1994.

[30] K. Lu, "An efficient method for analysis of arbitrary nonuniform transmission lines," IEEE Trans. Microw. Theory Techn., vol. 45, no. 1, pp. 9-14, Jan. 1997.
[31] A. O. Palusinski and A. Lee, "Analysis of transients in nonuniform and uniform multiconductor transmission lines," IEEE Trans. Microw. Theory Techn., vol. 37, no. 1, pp. 127-138, Jan. 1989.

[32] G.-W. Pan, G. J. Wunsch, and B. K. Gilbert, "Frequency-domain analysis of coupled nonuniform transmission lines using Chebyshev pseudospatial techniques," IEEE Trans. Microw. Theory Techn., vol. 40, no. 11, pp. 2025-2033, Nov. 1992.

[33] F.-Y. Chang, "Waveform relaxation analysis of nonuniform lossy transmission lines characterized with frequency-dependent parameters," IEEE Trans. Circuits Syst., vol. 38, no. 12, pp. 1484-1500, Dec. 1991.

[34] N. Orhanovic, P. Wang, and V. K. Tripathi, "Time-domain simulation of uniform and nonuniform multiconductor lossy lines by the method of characteristics," IEEE Trans. Comput.-Aided Des. Integr. Circuits Syst., vol. 12, no. 6, pp. 900-904, Jun. 1993.

[35] A. Khebir, A. B. Kouki, and F. M. Ghannouchi, "A pseudothreedimensional finite element analysis of nonuniform multiconductor transmission lines in the quasi-static and high-frequency regimes," IEEE Trans. Microw. Theory Techn., vol. 42, no. 12, pp. 2386-2390, Dec. 1994.

[36] D. Kuznetsov, "Efficient circuit simulation of nonuniform transmission lines," IEEE Trans. Microw. Theory Techn., vol. 46, no. 5, pp. 546-550, May 1998.

[37] S. Barmada and M. Raugi, "Transient numerical solutions of nonuniform MTL equations with nonlinear loads by wavelet expansion in time or space domain," IEEE Trans. Circuits Syst. I, Fundam. Theory Appl., vol. 47, no. 8, pp. 1178-1190, Aug. 2000.

[38] S. Grivet-Talocia, "Adaptive transient solution of nonuniform multiconductor transmission lines using wavelets," IEEE Trans. Antennas Propag., vol. 48, no. 10 , pp. 1563-1573, Oct. 2000.

[39] Q. Xu and P. Mazumder, "Accurate modeling of lossy nonuniform transmission lines by using differential quadrature methods," IEEE Trans. Microw. Theory Techn., vol. 50, no. 10, pp. 2233-2246, Oct. 2002.

[40] E. Gad and M. Nakhla, "Efficient simulation of nonuniform transmission lines using integrated congruence transform," IEEE Trans. Very Large Scale Integr. (VLSI) Syst., vol. 12, no. 12, pp. 1307-1320, Dec. 2004.

[41] M. Khalaj-Amirhosseini, "Analysis of nonuniform transmission lines using the equivalent sources," Progress in Electromagnetics Research, vol. 71, pp. 95-107, 2007.

[42] M. Chernobryvko, D. Vande Ginste, and D. De Zutter, "A two-step perturbation technique for nonuniform single and differential lines," IEEE Trans. Microw. Theory Techn., vol. 61, no. 5, pp. 1758-1767, May 2013.

[43] M. Chernobryvko, D. De Zutter, and D. Vande Ginste, "Nonuniform multiconductor transmission line analysis by a two-step perturbation technique," IEEE Trans. Compon. Packag. Manuf. Techol., vol. 4, no. 11 pp. 1838-1846, Nov. 2014.

[44] P. Manfredi, D. Vande Ginste, and D. De Zutter, "An effective modeling framework for the analysis of interconnects subject to line-edge roughness," IEEE Microw. Wireless Compon. Lett., vol. 25, no. 8, pp. 502-504, Aug. 2015.

[45] T. Dhaene, L. Martens, and D. De Zutter, "Transient simulation of arbitrary nonuniform interconnection structures characterized by scattering parameters," IEEE Trans. Circuits Syst. I, Fundam. Theory Appl., vol. 39 , no. 11, pp. 928-937, Nov. 1992.

[46] F. Grassi, Y. Yang, X. Wu, G. Spadacini, and S. A. Pignari, "On mode conversion in geometrically unbalanced differential lines and its analogy with crosstalk," IEEE Trans. Electromagn. Compat., vol. 57, no. 2, pp. 283-291, Apr. 2015.

[47] J .C. Clements, C. R. Paul, and A. T. Adams, "Computation of the capacitance matrix for systems of dielectric-coated cylindrical conductors," IEEE Trans. Electromagn. Compat., vol. EMC-17, no. 4, pp. 238-248, Nov. 1975.

[48] C. R. Paul and A. E. Feather, "Computation of the transmission line inductance and capacitance matrices from the generalized capacitance matrix," IEEE Trans. Electromagn. Compat., vol. EMC-18, no. 4, pp. 175183, Nov. 1976 


\begin{tabular}{|c|} 
\\
\\
PLACE \\
PHOTO \\
HERE \\
\end{tabular}

Paolo Manfredi (S'10-M'14) was born in 1985. He received the M.Sc. degree in electronic engineering from the Politecnico di Torino, Turin, Italy, in 2009 , and the Ph.D. degree in information and communication technology from the Scuola Interpolitecnica di Dottorato, Politecnico di Torino, in 2013.

He is currently a Postdoctoral Research Fellow with the Found for Scientific Research - Flanders (FWO), Electromagnetics Group, Department of Information Technology, Ghent University, Ghent, Belgium. In June 2011 and in February and March 2012, he was a Visiting Scientist with the Center for Advanced Life Cycle Engineering (CALCE), University of Maryland at College Park, MD, USA. From April 2012 to July 2012 and in May and June 2013, he was a Visiting Scientist with the Department of Information Technology, Ghent University. From March 2013 to September 2014, he was a Postdoctoral Researcher with the EMC Group, Department of Electronics and Telecommunications, Politecnico di Torino. His research interests comprise several aspects of circuit and interconnect modeling and simulation, including statistical and worst-case analysis, signal integrity, and electromagnetic compatibility. He authored or co-authored over 50 publications in international journals and conferences proceedings. He regularly serves as a reviewer for several international journals. He is the Program Co-Chair of the 20th IEEE Workshop on Signal and Power Integrity (SPI 2016).

Dr. Manfredi was selected for the IBM Best Student Recognition Event in 2009. He was the recipient of the Best Student Paper Award and the Best Oral Paper Award at the 19th and 22nd IEEE Conference on Electrical Performance of Electronic Packaging and Systems (EPEPS) in 2010 and 2013 , respectively, and of an Honorable Mention from the IEEE Microwave Theory and Techniques Society (MTT-S) International Microwave Symposium (IMS) in 2011. He was also the recipient of a Young Scientist Award at the XXX General Assembly and Scientific Symposium of the International Union of Radio Science (URSI GASS) in 2011

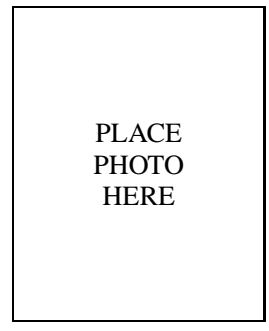

Dries Vande Ginste (SM'12) received the M.Sc. degree and the Ph.D. degree in electrical engineering from Ghent University, Gent, Belgium, in 2000 and 2005, respectively. He is currently an Associate Professor with the Department of Information Technology, Ghent University and a Guest Professor at iMinds. In June and July 2004, he was a Visiting Scientist at the Department of Electrical and Computer Engineering, University of Illinois at UrbanaChampaign (UIUC), IL, USA. From September to November 2011, he was a Visiting Professor at the EMC Group, Dipartimento di Elettronica, Politecnico di Torino, Italy. $\mathrm{He}$ has authored or co-authored over 100 papers in international journals and in conference proceedings. His research interests include computational electromagnetics, electromagnetic compatibility, signal and power integrity, and antenna design.

Dr. Vande Ginste was awarded the International Union of Radio Science (URSI) Young Scientist Award at the 2011 URSI General Assembly and Scientific Symposium, the Best Poster Paper Award at the 2012 IEEE Electrical Design of Advanced Packaging and Systems Symposium (EDAPS), the Best Paper Award at the 2013 IEEE Workshop on Signal and Power Integrity (SPI) and the Best Paper Award at the 2013 IEEE International Conference on Electrical Performance of Electronic Packaging and Systems (EPEPS). He served as the co-chair of the 2014 IEEE Workshop on Signal and Power Integrity. He is a Senior Member of the IEEE.

\begin{tabular}{|c|} 
\\
\\
PLACE \\
PHOTO \\
HERE \\
\end{tabular}

Daniël De Zutter (F'00) was born in 1953. He received his M.Sc. degree in electrical engineering from the University of Gent in 1976. In 1981 he obtained a Ph.D. degree and in 1984 he completed a thesis leading to a degree equivalent to the French Aggrégation or the German Habilitation. He is now a full professor of electromagnetics. His research focusses on all aspects of circuit and electromagnetic modelling of high-speed and high-frequency interconnections and packaging, on Electromagnetic Compatibility (EMC) and numerical solutions of Maxwell's equations. As author or co-author he has contributed to more than 200 international journal papers (cited in the Web of Science) and 200 papers in conference proceedings. In 2000 he was elected to the grade of Fellow of the IEEE. He was an Associate Editor for the IEEE Microwave Theory and Techniques Transactions. Between 2004 and 2008 he served as the Dean of the Faculty of Engineering of Ghent University and is now the head of the Department of Information Technology. 